\title{
O PESSEGUEIRO NO SISTEMA DE POMAR COMPACTO: VI. FRUTIFICAÇÃO EFETIVA E RALEIO QUÍMICO EM SELEÇÕES IAC $\left({ }^{1}\right)$
}

\author{
WILSON BARBOSA (24); FERNANDO ANTONIO CAMPO-DALL'ORTO (24); \\ MÁRIO OJIMA ( $\left.{ }^{2}\right)$ e RUI RIBEIRO DOS SANTOS ( $\left.{ }^{3}\right)$
}

\begin{abstract}
RESUMO
Verificou-se o potencial de frutificação e o efeito da pulverização de uréia a $12 \%$, no raleio de frutos, em cinco pessegueiros e duas nectarineiras, cultivados em pomar compacto, na Estação Experimental de Monte Alegre do Sul (22 $41^{\prime}$ S. e $46^{\circ} 43^{\prime} \mathrm{W}$.), do Instituto Agronómico de Campinas (IAC). Dos cultivares e seleçסes pesquisados, 'Talismã', IAC 6782-83, IAC N 2680-91, 'Aurora-2' e IAC 282-24 apresentaram as maiores taxas de frutificação natural, a saber: 60,$9 ; 54,2 ; 44,6 ; 41,4$ e $40,0 \%$ respectivamente. A uréia a $12 \%$, pulverizada na plena floraçăo, mostrou-se efetiva no raleio dos frutos, reduzinđo as frutificaçōes para a faixa de $12-17 \%$, considerada adequada para pessegueiros e nectarineiras sob altas densidades de plantio. O número médio de pêssegos e nectarinas remanescentes por ramo foi, respectivamente, de 1,$1 ; 1,6 ; 1,8 ; 1,9 ; 2,0 ; 2,6$ e 3,2 para IAC 280-28; IAC 6782-83; 'Aurora-2'; IAC 282-24; IAC N 1880-76; IAC N 2680-91 e Talismã'. A produtividade não foi prejudicada pela ação raleadora da uréia a $12 \%$. As plantas em que permaneceram até dois frutos em média, por ramo, apresentaram produtos de melhor padrão.
\end{abstract}

Termos de indexação: pêssego, Prunus persica (L.) Batsch; nectarina, Prunus persica (L.) Batsch, var. nucipersica, frutificação, raleio químico, uréia, pomar compacto.

\section{ABSTRACT \\ THE PEACH MEADOW ORCHARD SYSTEM: VI. FRUIT SET AND CHEMICAL THINNING IN IAC SUBTROPICAL SELECTIONS}

This paper reports the fruit set potential and effects of $12 \%$ urea on fruit drop in peach and nectarine selections in meadow orchard (5,000 plants per hectare). The experimental plot was located at the Estação Experimental of Monte Alegre do Sul ( $22^{\circ} 41$ 'S. and $46^{\circ} 43^{\prime} \mathrm{W}$.) of the Instituto Agronomico (IAC), State of São Paulo, Brazil, which has a record of 40 hours per year with temperature bellow $7^{\circ} \mathrm{C}$. The best fruit set was obtained by 'Talismã', IAC 6782-83, LAC N 2680-91, 'Aurora-2' and LAC $282-24$, showing $60.9,54.2,44.6,41.4$ and $40.0 \%$ respectivelly. The urea $12 \%$, sprayed at full bloom stage, significantly decreased fruit set to $12-17 \%$. The average fruit number remaining per branch was, respectivelly: 1.1, 1.6, 1.8, 1.9, 2.0, 2.6 and 3.2 for IAC 280-28, IAC 6782-83, 'Aurora-2', IAC 282-24, IAC N 1880-76, IAC N 2680-91 and 'Talismä'. The selections which held up to two fruits per branch showed better products.

Index terms: peach, Prunus persica (L.) Batsch; nectarine, Prunus persica (L.) Batsch, var. nucipersica; fruit set, fruit drop, chemical thinning, urea, meadow orchard.

(1) Pesquisa executada com recursos parciais do CNPq, dentro do Projeto IAC: "Melhoramento genético e cultural do pessegueiro e da nectarineira". Recebido para publicaçāo em 5 de agosto e aceito $\mathrm{em} 30$ de setembro de 1991.

$\left(^{2}\right)$ Seçāo de Fruticultura de Clima Temperado, Instituto Agronômico (IAC), Caixa Postal 28, 13001-970 Campinas (SP).

(3) Estação Experimental de Monte Alegre do Sul (IAC).

$\left.{ }^{4}\right)$ Com bolsa de pesquisa do CNPq. 
Os pessegueiros e as nectarineiras adaptadas ao clima paulista de pouco frio hibernal apresentam, anualmente, elevado índice de frutificação efetiva. Quando cultivados em sistema de pomar compacto, fixam quantidade de frutos até dez vezes superior a sua capacidade ideal de suporte. Esses frutos, se mantidos todos nas plantas, desenvolvem-se insuficientemente e se tornam inviáveis à comercialização; daí, a necessidade da prática do raleio, seja manual ou mecânica, seja química.

Em relação ao raleio químico de frutas de caroço, observa-se consistente avanço de pesquisas que se utilizam de reguladores de crescimento, dessecantes, fertilizantes, inibidores fotossintéticos e agentes fitotóxicos. Entre os produtos químicos já experimentados, destacaram-se os seguintes: ácido 2-cloroetil fosfônico - Ethephon (Buchanan \& Biggs, 1969; Stembridge \& Gambrell Júnior, 1971; Weinbaum et al., 1977; Blanco, 1987); ácido naftalenacético (ANA) (Kelly, 1955; Lombard \& Mitchell, 1962; Feliciano et al., 1978); 2-(3 clorofenoxi-) - propiônico - 3 CPA (Beutel et al., 1969; Martin \& Nelson, 1969; Morini et al., 1976); beta-cloroetil-metil-bis-benziloxi-silane (CGA) (Byers, 1978; Gambrell Júnior et al., 1983), e compostos nitrogenados como uréia e nitrato de amônio (Erez, 1975; Byers \& Lyons Júnior, 1985; Zilkah et al., 1988). Desses produtos, a uréia, nas concentrações entre 8 e $12 \%$, quando pulverizada em floradas de pessegueiros e nectarineiras, reduziu a frutificação aos níveis desejáveis e proporcionou adequada qualidade organoléptica aos frutos, segundo Zilkah et al. (1988). Esses autores atentam à inofensividade da uréia no raleamento de frutos, por ser esta um constituinte natural da planta e do homem. Em contraposição, ressaltam que determinados produtos sintéticos poderiam provocar distúrbios físicos à planta e malefícios à saude humana.

No Brasil, são escassas as pesquisas sobre o raleio químico de frutos de pessegueiros e nectarineiras sob cultivo convencional $e$, ainda, inexistentes se em altas densidades populacionais (Castro et al., 1989). Em face disto, pesquisou-se o efeito da uréia no raleio e o potencial de frutificação efetiva em seleçōes IAC de pêssegos e de nectarinas subtropicais, cultivadas no sistema de pomar compacto (Barbosa, 1989; Barbosa et al., 1989).

\section{Material e Métodos}

Utilizaram-se na experimentação pessegueiros e nectarineiras de quatro anos, cultivados no espaçamento de 4 x 0,5m (5.000 plantas/hectare), na Estação Experimental de Monte Alegre do Sul (22 ${ }^{\circ} 41^{\prime}$ S.; $46^{\circ} 49^{\prime} \mathrm{W}$. e $\left.40 \mathrm{HF}-7\right)\left({ }^{5}\right)$, do IAC (Pedro Júnior et al., 1979). Os cultivares e seleções utilizados: pêssego - Talismã, Aurora-2, IAC 6782-83, IAC 280-28 e IAC 282-24, e nectarinas - IAC N 1880-76 e IAC N 2680-91, receberam os tratos culturais recomendados (Ojima et al., 1990), à exceção da poda semidrástica (Barbosa et al., 1990).

No início de julho de 1990, pulverizaram-se as plantas, em plena floração, com uréia na concentração de $12 \%$, acrescida de espalhante adesivo a $0,02 \%$. Para cada cultivar e seleção, utilizaram-se aleatoriamente cinco plantas; os ramos, em número de dez por planta, foram tomados ao acaso e identificados com etiquetas, anotando-se nestas o número total de flores abertas, sendo os botōes florais eliminados dos ramos. Controlaram-se aproximadamente 600 flores por material. Para as cinco plantas testemunhas, sem pulverização com uréia $12 \%$, adotou-se o mesmo método de identificação e de controle dos ramos c flores. Após $\mathbf{4 0}$ dias da antese, controlaram-se, para cada material e tratamento, os frutos remanescentes nos ramos, estabelecendo-se, daí, os índices médios de frutificação efetiva. Nas plantas testemunhas, após o controle da frutificação efetiva natural, executou-se o raleio manual, deixando-se um e dois frutos por ramo médio e vigoroso respectivamente; nos ramos finos, o raleio foi total. De fin $s$ de setembro a meados de novembro do mesmo ano, os frutos maduros foram colhidos, contados e pesados.

Aos dados da frutificação efetiva, do número de frutos por ramo, da produção e do peso final dos pêssegos e das nectarinas, calcularam-se os intervalos de confiança para a média, ao nível de $95 \%$.

(5) Latitude, longitude e número médio de horas anuais de frio abaixo de $7^{\circ} \mathrm{C}$ (HF-7) respectivamente. 


\section{Resultados e Discussão}

Os pessegueiros e as nectarineiras testemunhas apresentaram índices de abscisão de flores e frutos entre 40 e $70 \%$, considerados normais para seleçōes autoférteis, adaptadas ao clima subtropical-tropical paulista (Barbosa et al., 1991). Suas frutificações efetivas naturais apresentaram-se altas, sendo o 'Talismã', IAC 6782-83, IAC N 2680-91, 'Aurora-2' e IAC 282-24 os mais prolíferos, com 60,$9 ; 54,2 ; 44,6 ; 41,4$ e $40,0 \%$ respectivamente (Figura 1).

A abscisão de flores e de frutinhos, que se iniciou após uma semana da pulverização com uréia $12 \%$, persistiu até cerca de $\mathbf{4 0}$ dias da antese. Verificou-se, no entanto, maior intensidade de abscisão durante a $2^{\mathrm{a}}$ e a $3^{\mathrm{a}}$ semana pós-tratamento das flores. Byers \& Lyons Junior (1985), utilizando-se de compostos nitrogenados, a exemplo de nitrato de amônio
$\left(\mathrm{NH}_{4} \mathrm{NO}_{3}\right)$, como agente raleador, notaram aparecimento de necroses florais, cerca de dois dias após aplicação do produto. Conjecturaram que o $\mathrm{NH}_{4} \mathrm{NO}_{3}$ poderia agir de dois modos: interferindo na fertilizaçāo da oosfera e necrosando os pedúnculos da maioria dos botões florais e das flores em antese. A presença das necroses no pedúnculo seria, então, resultado da translocaçāo e da concentração dessas substâncias em proporções fitotóxicas.

As flores e os frutos em crescimento, de todo o material experimentado, mostraram-se bastante sensíveis à ação da uréia a $12 \%$, porém em graus diferentes. No 'Talismã' e na IAC N 2680-91, reduziu-se intensamente a frutificação efetiva; por se tratar, no entanto, de pessegueiro e de nectarineira altamente floríferos e férteis, ainda permaneceram com 3,2 e 2,6 frutos, em média, por ramo respectivamente (Figura 1).

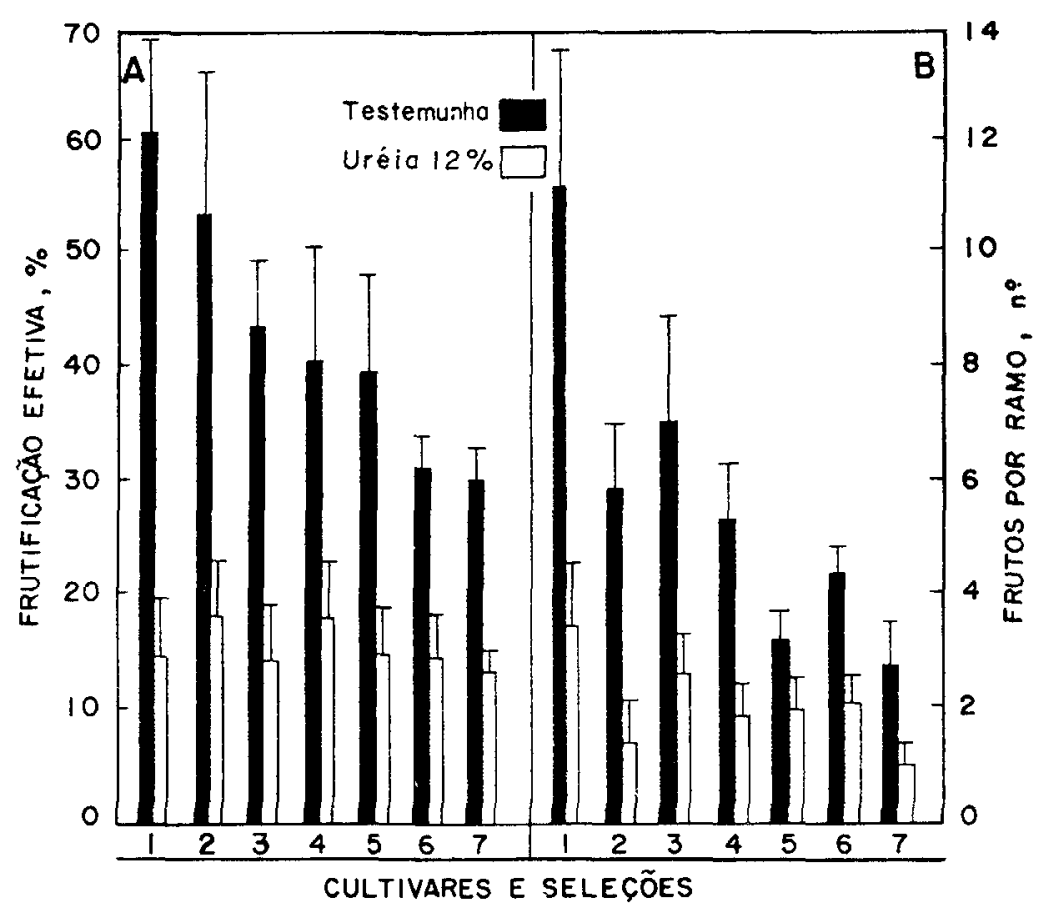

Figura 1. Efeito da uréia $12 \%$, pulverizada na florada. A: na porcentagem de frutificação efetiva; B: no número de frutos por ramo de pessegueiros e de nectarineiras (N) IAC, cultivados no sistema de pomar compacto (5.000 plantas/hectare), na Estação Experimental de Monte Alegre do Sul. 1. 'Talismā'; 2: IAC 6782-83; 3: IAC N 2680-91; 4: 'Aurora-2'; 5: IAC 282-24; 6: IAC N 1880-76; 7: IAC 280-28. As linhas verticais sobre as colunas indicam o desvio-padrão da média, ao nível de $5 \%$. 
Com essa quantidade de frutos remanescentes por ramo, o peso médio do produto final apresentou-se aquém do esperado (Figura 2), problema esse que poderia ser contornado com raleios manuais corretivos. Sabe-se, outrossim, que a uréia pulverizada nas flores, em altas concentrações, pode promover excessivo raleamento, diminuindo significativamente a produção de frutos. Na nectarineira 'Rubro-sol', concentrações entre 12 e $16 \%$ de uréia causaram fitotoxicidade em ramos jovens e queda exagerada de frutos; apesar do aumento do peso médio das nectarinas, proporcionado pela menor competição de nutrientes, a produtividade não foi compensadora (Zilkah et al., 1988).

Os pessegueiros e as nectarineiras IAC, após cerca de $\mathbf{4 0}$ dias do tratamento com uréia $12 \%$, mantiveram-se com frutificaçōes efetivas na faixa de 12-17\%. Excetuando o 'Talismã' e a IAC N 2680-91, os cultivares e seleçōes permaneceram com um a dois frutos por ramo, quantidade considerada ade- quada para cultivos em pomar compacţo. Nestes, a produção e o peso médio dos frutos apresentaram-se similares aos das respectivas plantas testemunhas, com raleio manual (Figura 2).

É importante destacar que pessegueiros e nectarineiras, sob altas densidades de plantio, tendem a apresentar frutos com pesos médios $20 \%$ inferiores, em confronto aos dos cultivos convencionais (BARBOSA et al., 1991). A uréia a 12\%, como nutriente vegetal, não influenciou na qualidade final dos frutos, que permaneceram nos níveis característicos dos cultivares e das seleçōes em estudo.

Convém lembrar, finalmente, que em face dos diferentes níveis varietais de sensibilidade das flores à uréia, são necessários novos experimentos, sob condições edafoclimáticas diversas. Assim, concentrações ideais de uréia deverão ser pesquisadas, para cada material, objetivando adequado raleio químico que compense produção econômica de pêssegos e nectarinas, cultivados em pomar compacto.

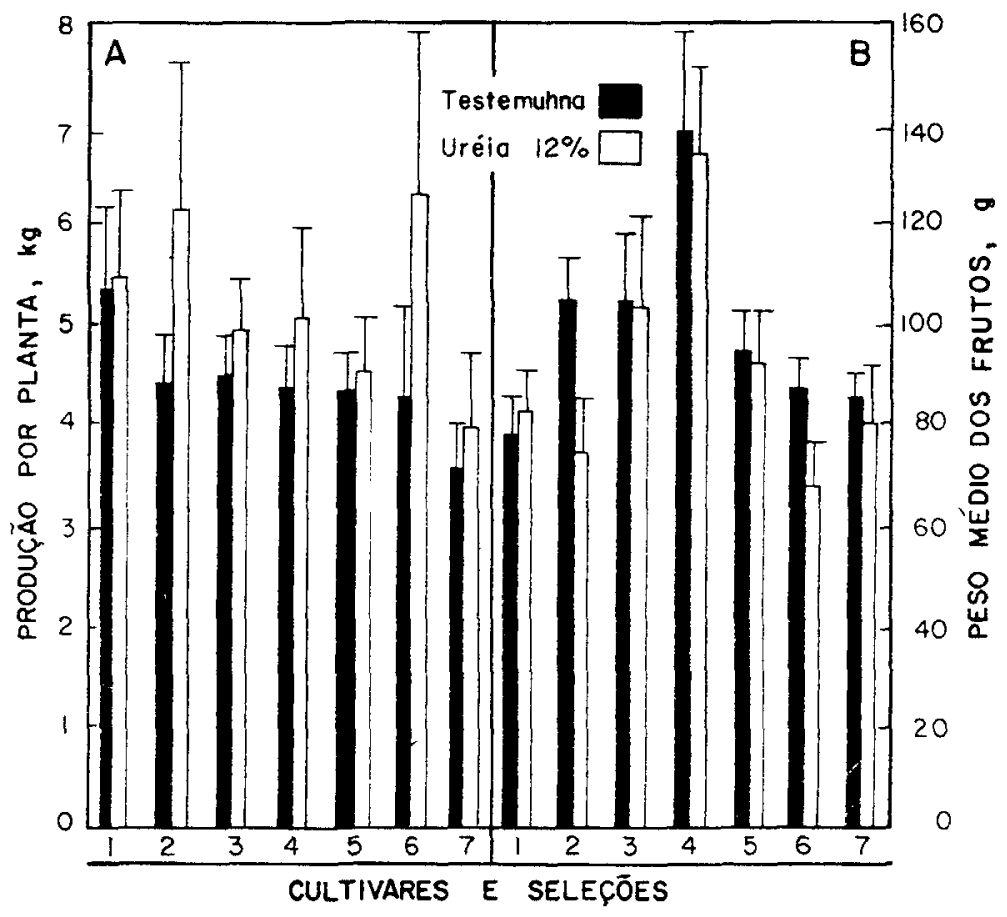

Figura 2. Efeito da uréia $12 \%$, pulverizada na florada. A: na produção; B: no peso final dos frutos de pessegueiros e de nectarineiras (N) IAC, cultivados no sistema de pomar compacto (5.000 plantas/hectare), na Estação Experimental de Monte Alegre do Sul. 1: 'Aurora-2'; 2: 'Talismā'; 3: IAC 282-24; 4: IAC 6782-83; 5: IAC 280-28; 6: IAC N 2680-91; 7: IAC N 1880-76. Testemunha = raleio manual. As linhas verticais sobre as colunas indicam o desvio-padrão da média, ao nível de $5 \%$. 


\section{AGRADECIMENTO}

Os autores agradecem ao Auxiliar de Campo Lázaro de Godói os tratos culturais dos pessegueiros e das nectarineiras do experimento.

\section{REFERÊNCIAS BIBLIOGRÁFICAS}

BARBOSA, W. Desenvolvimento vegetativo e reprodutivo do pessegueiro em pomar compacto sob poda drástica anual. Piracicaba, 1989. 154p. Dissertaçāo (Mestrado em Agronomia) - ESALQ-USP, 1989.

BARBOSA, W.; CAMPO-DALL'ORTO, F.A. \& OIMA, M. O pessegueiro no sistema de pomar compacto: I. Conjeturas, experimentaçāo e prática. O Agronômico, Campinas, 41(1):26$-39,1989$.

BARBOSA, W.; CAMPO-DALL'ORTO, F.A.\& OJIMA, M. O pessegueiro no sistema de pomar compacto: V. Pesquisas do Instituto Agronômico na década de 80. O Agronômico, Campinas, 42(1):35-44, 1990.

BARBOSA, W.; CAMPO DALL'ORTO, F.A. \& OJIMA, M. Conservação e germinação do pólen, polinização e frutificação efetiva em pessegueiro e nectarineiras subtropicais. Campinas, Instituto Agronômico, 1991. 21p. (Datilografado)

BARBOSA, W.; CAMPO-DALL'ORTO, F.A.; OJIMA, M.; MARTINS, F.P. \& IGUE, T. O pessegueiro no sistema de pomar compacto: IV. Intensidade e época de raleio dos frutos dos cultivares 'Tropical' e 'Aurora-1'. Bragantia, Campinas, 50(1):93-102, 1991.

BEUTEL, J.; YEAGER, J.; POST, G.; ROUGH, D.; ANDERSON, W.; ROSS, N.; PERRY, F.; GERDTS, M.; LA RUE, J. \& BROWN, L. Cling peaches effectively thinned with 3-CPA. California Agriculture, Berkeley, 23(2):6-8, 1969.

BLANCO, A. Efecto del ethephon sobre la caida y maduracion del fruto en melocotonero y nectarina. Información Técnica Económica Agraria, Zaragoza, 73:3-14, 1987.

BUCHANAN, D.W. \& BIGGS, R.H. Peach fruit abscission and pollen germination as influenced by ethylene and 2-chloroethane phosphonic acid. Journal of the American Society for Horticultural Science, Greensboro, 94:327-329, 1969.

BYERS, R.E. Chemical thinning of peach fruit with CGA 15281 and CGA 17856. Journal of the American Society for Horticultural Science, Mount Vernon, 103:232-236, 1978.

BYERS, R.E. \& LYONS JUNIOR, C.G. Peach flower thinning and possible sites of action of desiccating chemicals. Journal of the American Society for Horticultural Science, Alexandria, 110:662-667, 1985 .

CASTRO, M.L.N.D.; NEVES, R.M.R.B. \& TERRA, M.M. Contribuiçäo à bibliografia nacional de fruticultura (1970-1987). Campinas, Sociedade Brasileira de Fruticultura, 1989. 485p.
EREZ, A. Thiourea, a new thinning agent for early ripening peaches and nectarines. HortScience, Alexandria, 10:251-253, 1975.

FELICIANO, A.J.; NAKASU, B.H. \& MAGNANI, M. Raleio químico em pessegueiro cultivar Capdebosq. In: CONGRESSO BRASIL.EIRO DE FRUTICULTURA, 4., Salvador, 1977. Anais. Cruz das Almas, Sociedade Brasileira de Fruticultura, 1978. p.283-290.

GAMBRELL JUNIOR, C.E.; COSTON, D.C. \& SIMS JUNIOR, E.T. Results of eigth years with CGA-15281 as a postbloom thinner for peaches. Journal of the American Society for Horticultural Science, Alexandria, 108:605-608, 1983.

KELLY, V.W. Time of application of naphthaleneacetic acid for fruit thinning of the peach in relation to june drop. Proceedings of the American Society for Horticultural Science, Geneva, 66:70-72, 1955 .

LOMBARD, P.B. \& MTTCHELL, A.E. Anatomical and hormonal development of Redhaven peach seeds as related to the timing of naphthaleneacetic acid for fruit thinning. Proceedings of the American Society for Horticultural Science, New York, 80:163-171, 1962.

MARTIN, G.C. \& NELSON, M. The thinning effect of 3-chlorophenoxipropionamide (3-CPA) in Paloro peach. Hort Science, Alexandria, 4:106-108, 1969.

MORINI, S.; VITAGLIANO, C. \& XILOYANNIS, C. Effects of 3-CPA and ethephon on growth and abscission of 'Cardinal' peach. Journal of the American Society for Horticultural Science, Mount Vemon, 101:640-642, 1976.

OJIMA, M.; CAMPO-DALL'ORTO, F.A.; BARBOSA, W. \& RIGITANO, O. Pêssego (Prunus persica Batsch). In: INSTTTUTO AGRONÔMICO (Campinas). Instruçōes Agrícolas para o Estado de São Paulo. 5.ed. Campinas, 1990. p. 170-171. (Boletim, 200)

PEDRO JÚNIOR, M.J.; ORTOLANI, A.A.; RIGITANO, O.; ALFONSI, R.R.; PINTO, H.S. \& BRUNINI, O. Estimativa de horas de frio abaixo de 7 e de $13^{\circ} \mathrm{C}$ para regionalização da fruticultura de clima temperado no Estado de São Paulo. Bragantia, Campinas, 38:123-130, 1979.

STEMBRIDGE, G.E. \& GAMBRELL JUNIOR, C.E. Thinning peaches with bloom and postbloom applications of 2-chloroethyl-phosphonic acid. Journal of the American Society for Horticultural Science, La Jolla, 96:7-10, 1971.

WEINBAUM, S.A.; GIULIVO, C. \& RAMINA, A. Chemical thinning: ethylene and pre-treatment fruit size influence enlargement, auxin transport, and apparent sink strenght of French prune and 'Andross' peach. Journal of the American Society for Horticultural Science, Mount Vernon, 102:781$785,1977$.

ZILKAH, S.; KLEIN, I. \& DAVID, J. Thinning peaches and nectarines with urea. Journal of Horticultural Science, London, 63(2):209-216, 1988. 